\title{
Dielectric function of $\mathrm{CaF}_{2}$ between 10 and $35 \mathrm{eV}$
}

\author{
J. Barth, ${ }^{*}$ R. L. Johnson, ${ }^{\dagger}$ and M. Cardona \\ Max-Planck-Institut für Festkörperforschung, Heisenbergstrasse 1, D-7000 Stuttgart 80, Federal Republic of Germany \\ D. Fuchs and A. M. Bradshaw \\ Fritz-Haber-Institut der Max-Planck-Gesellschaft, Faradayweg 4-6, D-1000 Berlin 33, Federal Republic of Germany
}

(Received 29 November 1989)

\begin{abstract}
The dielectric function of $\mathrm{CaF}_{2}$ over the photon energy region 10-35 eV has been determined by spectroscopic ellipsometry. Close to the fundamental absorption edge the data agree fairly well with band-structure calculations. A sharp exciton at the Ca $3 p$ threshold $(27.9 \mathrm{eV})$ is immediately followed by structures which can be attributed to variations in the conduction-band density of states. The strong transitions observed at higher energies cannot be explained in the one-electron band-structure model. Detailed theoretical calculations are required.
\end{abstract}

Although the electronic band structure of semiconductors has been extensively investigated comparatively few studies have been performed to date on wide band-gap semiconductors and insulators because of experimental difficulties. Electron spectroscopic techniques are complicated by the charging of the samples, and to probe the electronic interband transitions optically one has to work in the vacuum-ultraviolet (vuv) spectral range. With the advent of synchrotron radiation, reflectance spectra of many insulators were measured and for some materials modulated reflectance spectra were also obtained.

In this paper we present the dielectric function of $\mathrm{CaF}_{2}$ measured with our newly developed vuv ellipsometer. ${ }^{1}$ The normal-incidence reflectance calculated from our ellipsometric dielectric function agrees well with our own direct measurements. We are convinced that our data are significantly more accurate than most of the previously obtained reflectance ${ }^{2-5}$ and energy-loss ${ }^{6}$ measurements which typically show discrepancies of up to a factor of 2 in either the magnitude of the reflectivity or in $\epsilon_{1}$ and/or $\epsilon_{2}$. The dielectric function between 11 and $27 \mathrm{eV}$ is discussed in terms of valence electron transitions using a recent band-structure calculation to assign the observed spectral features. Above $27 \mathrm{eV}$ excitations of the $\mathrm{Ca} 3 p$ core electrons dominate the spectrum with a sharp core exciton at threshold and the $3 p-3 d$ dipole resonance $4 \mathrm{eV}$ above.

The ellipsometer uses light from the 2-m SeyaNamioka monochromator at the synchrotron radiation laboratory Berliner Elektronenspeicherring-Gessellschaft für Synchrotronstrahlung $\mathrm{mbH}$ (BESSY), Berlin. Because of the high degree of linear polarization, ${ }^{1}$ the light is incident directly on the sample without a polarizer. To select the most appropriate angle between the plane of polarization and the plane of incidence the entire ellipsometer can be rotated about the axis of the incoming light. The angle of incidence was $45^{\circ}$ in the present experiment. Reflectance measurements at an angle of incidence of $2.5^{\circ}$ were also recorded in the same apparatus using a $\mathrm{GaP}$ photodiode detector. ${ }^{7}$ The sample was a $\mathrm{CaF}_{2}$ single crystal cleaved along the (111) plane at a pressure of $10^{-8}$ mbar.

Figure 1 shows the reflectance of $\mathrm{CaF}_{2}$ at room temper- ature. The observed structures are due to interband excitations between the $\mathrm{F}^{-} 2 p$-derived valence and the $\mathrm{Ca}^{2+}$ $4 s$ - and $3 d$-derived conduction bands. The normal incidence reflectance calculated from the ellipsometrically determined dielectric function for the same surface is also shown in Fig. 1. The calculated values are in good agreement with the directly measured reflectance. We note that the reflectivity data of Refs. 3 and 6 are in acceptable agreement with our data, while those of Refs. 4 and 5 yield two high values of the reflectivity at the peaks at 14 and $15.6 \mathrm{eV}$. All structures observed here are, however, also seen in the other measurements. At low photon energies the spectra are distorted by second-order light from the monochromator. Below $10.7 \mathrm{eV}$ the second-order light can be completely suppressed by using a $\mathrm{MgF}_{2}$ window. Results between 10.7 and $12 \mathrm{eV}$ are obtained by interpolation. Note that the first exciton has been placed at $11.2 \mathrm{eV}$ (Ref. 2) in the region where our data have poor

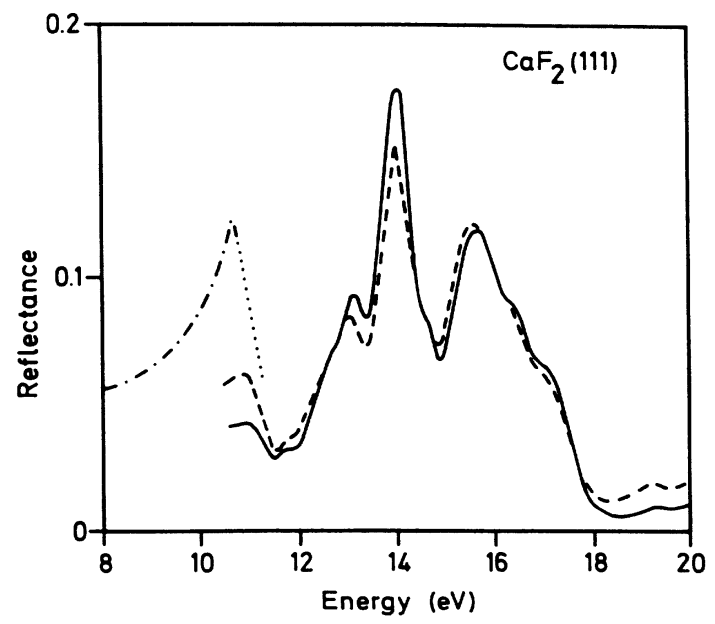

FIG. 1. The reflectance of $\mathrm{CaF}_{2}$ at room temperature: solid line, calculated from the ellipsometrically obtained dielectric function; dashed line, direct measurement; dash-dotted line, direct measurement behind a $\mathbf{M g F}_{2}$ filter to eliminate secondorder radiation. The dots indicate the most reasonable interpolation between both sets of data. 


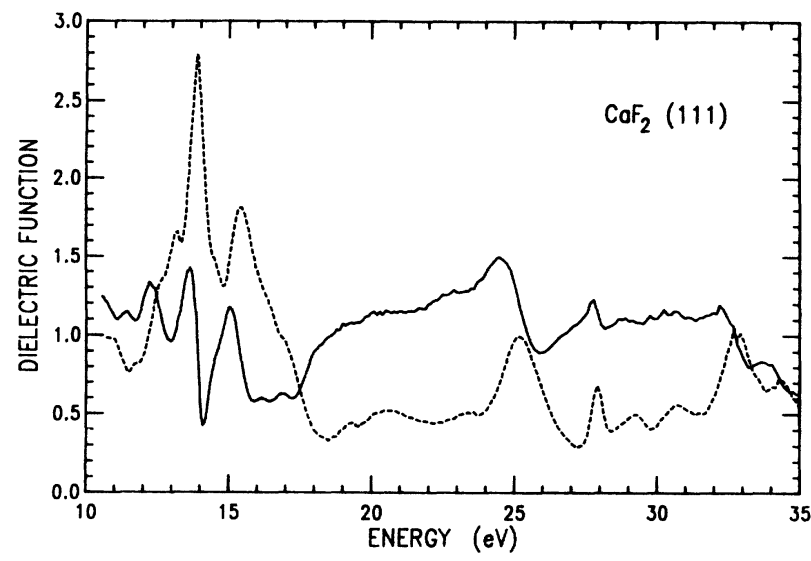

FIG. 2. The dielectric function of $\mathrm{CaF}_{2}$ at room temperature: solid line, real part; dashed line, imaginary part.

accuracy because of contamination with second-order radiation.

Figure 2 shows the dielectric function of $\mathrm{CaF}_{2}$ at room temperature up to $35 \mathrm{eV}$. Considerable structure is observed throughout the entire energy range, most of which can also be seen in the data of Refs. 3, 4, and 6. To simplify the discussion we will divide the spectrum into two sections: the valence transitions up to $27 \mathrm{eV}$, and the $\mathrm{Ca}$ $3 p$ core excitations which extend from 27 to $35 \mathrm{eV}$.

The $\mathrm{CaF}_{2}$ sample was cooled to $90 \mathrm{~K}$ in order to resolve the details better in the low-energy region. The corre-

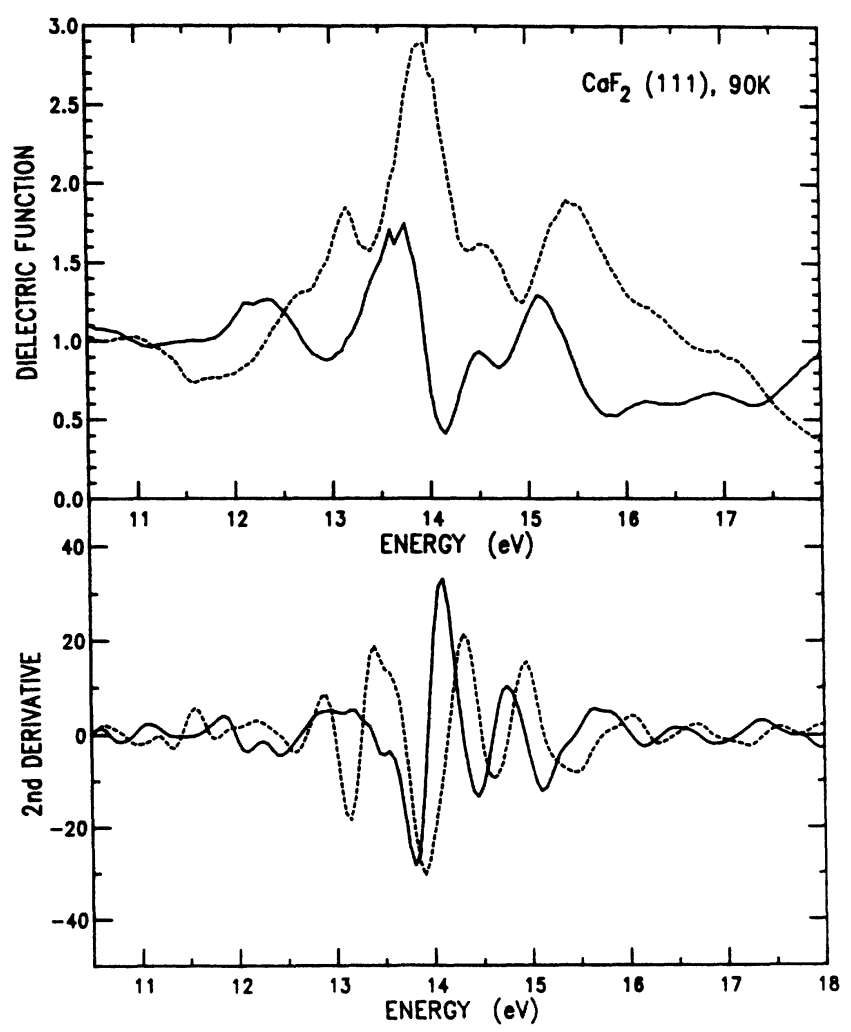

FIG. 3. Dielectric function at $90 \mathrm{~K}$ (upper panel) and its numerically obtained second energy derivative (lower panel); solid line, real part; dashed line, imaginary part. sponding real and imaginary components of the dielectric function are displayed in Fig. 3 along with the numerically obtained second derivatives with respect to photon energy. The line shapes of all prominent features seem to reflect excitonic effects, but a more detailed line-shape analysis is difficult because of the many overlapping structures. This is due to the complicated conduction-band structure of $\mathrm{CaF}_{2}$ where the $\mathrm{Ca} 3 d$ - and $4 s$-derived bands overlap near the bottom of the conduction band.

At present there are differing opinions about several important features of the $\mathrm{CaF}_{2}$ band structure. Here we will apply the band structure presented by Heaton and $\mathrm{Lin}^{8}$ to interpret the experimental data tentatively. Heaton and Lin used a self-consistent linear combination of atomic orbitals (LCAO) method to calculate the band structure. In these calculations exchange was treated in the localdensity approximation. If the correct Kohn-Sham exchange functional is used it is well known that, in general, excitation gaps too small compared with the experimental values are found. 9,10 In Ref. 8 this problem is overcome by using instead the Slater form of the exchange potential which corresponds to Kohn and Sham's multiplied by $\frac{4}{3}$. Although still a rough approximation, it is known that this leads to better values for the energies of excitation gaps. We thus believe that the bands of Ref. 8 are a good starting point for the discussion of the experiments. In Fig. 4 the calculated joint density of states (JDOS) from Ref. 8 is compared to the imaginary part of the dielectric function of Fig. 3. In order to align the structures we had to shift the calculated JDOS by $1.5 \mathrm{eV}$ towards higher energies. Such a shift was also applied by Heaton and Lin $^{8}$ when comparing their JDOS to published reflectance data. The agreement between calculation and experiment is quite good except for the high-energy side where the calculation places the peak labeled $D$ somewhat low in energy and does not reproduce its observed height and

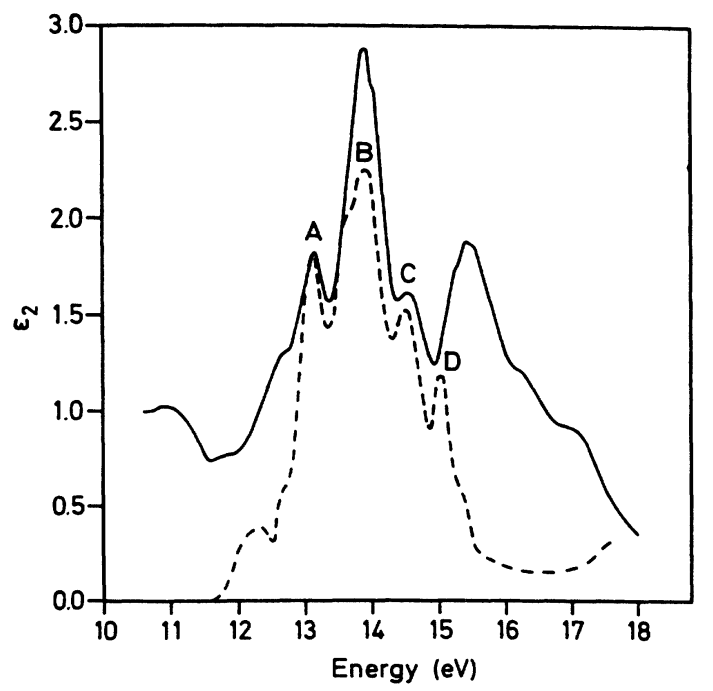

FIG. 4. Imaginary part (solid line) of the dielectric function of Fig. 3 compared to the calculated joint density of states (dashed) from Ref. 8. The vertical scale of the JDOS is arbitrary; its energy scale is rigidly shifted by $1.5 \mathrm{eV}$ towards higher energies. 
width. Hence, either the calculated band structure does not give the correct dispersion of the bands at higher energies or many-body effects (e.g., electron-hole interactions) introduce differences between JDOS and optical excitation spectra.

The prominent peaks in $\epsilon_{2}$ shown in Fig. 4 thus appear to originate mainly from transitions along $\Delta$ where the valence $p$ bands and the conduction $d$ bands are quite parallel. After going through a pronounced minimum near $18 \mathrm{eV}, \epsilon_{2}$ increases again at $19 \mathrm{eV}$ (see Fig. 2). Using Heaton and Lin's band structure ${ }^{8}$ as a guideline, we associate the following weak maxima at 19.5, 20.5, and $23.5 \mathrm{eV}$ with transitions into flat portions of the conduction bands at the edges of the Brillouin zone at $L$ and $X$ ( $L_{1 c}$ and $X_{4 c^{\prime}, 1 c}$ ). The strong maximum at $25.2 \mathrm{eV}$ with a weak sideband near $27 \mathrm{eV}$ may be attributed to transitions into a flat conduction band along $\Delta$ which the calculation places about $10 \mathrm{eV}$ above the bottom of the conduction band. $^{8}$ An earlier assignment ${ }^{2}$ of the corresponding reflectance structure to $\mathrm{F} 2 s$ excitations is contradicted by recent photoemission data ${ }^{11}$ which locates the $\mathrm{F} 2 s$ level at a binding energy about $3 \mathrm{eV}$ larger than the $\mathrm{Ca} 3 p$ level.

The above discussion accounts for the observed structures in terms of interband transitions. On the other hand, the first peak at $11.2 \mathrm{eV}$ (Ref. 2) has been interpreted as a valence exciton. Since the calculated threshold for direct transitions is $10.0 \mathrm{eV}$ (Ref. 8) one obtains, with the empirical 1.5-eV shift used in Fig. 4, an exciton binding energy of $0.3 \mathrm{eV}$. There is a clear steplike structure observed at $11.6 \mathrm{eV}$ and associating this with the first interband transition at $\Gamma$ we find an exciton binding energy of $0.4 \mathrm{eV}$ consistent with the one above, but differing from Rubloff, ${ }^{5}$ who identified the reflectance structure at 12.1 $\mathrm{eV}$ as the first interband transition. According to the JDOS the 12.1-eV structure must be attributed to higher critical points (see Fig. 4). Because of the excitonic line shapes of all the major structures observed in our dielectric function, all interband transitions are probably also down shifted by an excitonic interaction.

The structure at $27.9-\mathrm{eV}$ photon energy is considerably sharper than those just discussed. Its excitation energy is $16.7 \mathrm{eV}$ higher than that of the first valence exciton. This energy separation compares well with the binding energy difference of $17.6 \mathrm{eV}$ (Ref. 12) between the top of the valence band and the $\mathrm{Ca} 3 p$ core level obtained from photoemission data. Therefore, we identify it as the first $\mathrm{Ca}$ $3 p$ core exciton. Following it there is a series of structures at $29.2,30.6,32.8$, and $34.4 \mathrm{eV}$ (positions of maxima in $\left.\epsilon_{2}\right)$ all of which we assign to $\mathrm{Ca} 3 p \rightarrow 3 d$ and $3 p \rightarrow 4 s$ excitations because of their large oscillator strength. The $F$ $2 s$ excitations which are expected about $3 \mathrm{eV}$ above the Ca $3 p$ threshold $^{11}$ are not considered to be possible candidates because of their relatively small oscillator strength.

The imaginary part of the dielectric function corresponding to the Ca $3 p$ core exciton can be fitted by a single Gaussian peak centered at $27.93 \mathrm{eV}$ with a full width of $0.45 \mathrm{eV}$. There is no indication of the spin-orbit splitting of the $3 p$ core [0.35 eV (Ref. 13)] which should produce a clear asymmetry on the high-energy side of the exciton peak. In the case of $\mathrm{Mg}$ halides the $\mathrm{Mg} 2 p \rightarrow 3 s$ exciton excitation has been interpreted in terms of a multi- plet structure due to atomiclike coupling between the core hole and the excited electron. ${ }^{14}$ The dominant absorption peak has been assigned to the $\left(2 p^{5} 3 s\right){ }^{1} P_{1}$ final state and a weak shoulder at lower energy to the ${ }^{3} P_{1}$ configuration which becomes partly allowed by intermediate coupling. Adopting the same approach here we note that the ${ }^{3} P_{1}-{ }^{1} P_{1}$ splitting in $\mathrm{Ca}^{2+}$ is $0.5 \mathrm{eV} .{ }^{13}$ Within the range of uncertainty of this parameter it is possible to explain why the second peak related to the first core exciton is not observed. Hence, we consider plausible the assignment of this peak to transitions into a $3 p^{5} 4 s$ configuration. An admixture of $3 p^{5} 3 d$ cannot be excluded, but should be quite weak because of the low oscillator strength of these transitions at threshold. ${ }^{15}$

It is tempting to assign the oscillator strength observed above this exciton up to $31 \mathrm{eV}$ to the density of conduction states which start at the $X$ point. Since this density of states contains considerable $3 d$ components, the assignment just given requires neglect of configuration interaction in the $\left(3 p^{5} 3 d\right)$ final states. This is a rather unpalatable approximation since $\left(3 p^{5} 3 d\right)$ multiplet splittings of the order of $5 \mathrm{eV}$ are known to exist in atomic $\mathrm{Ca} .{ }^{15}$ Since these splitting energies are larger than the one-electron conduction-band widths under consideration, we do not believe the one-electron picture can be supported in the region where $\left(3 p^{5} 3 d\right)$ excitations are involved. This is with certainty the region above $30 \mathrm{eV}$, probably even lower. We thus believe that more detailed calculations, probably using an excited $\mathrm{Ca}$ atom as a center of a small $\mathrm{CaF}_{2}$ cluster, are required for the detailed interpretation of the $3 p$ to conduction-band transitions, in particular the strong peaks at 32.8 and $34.4 \mathrm{eV}$. The centrifugal barrier for the $3 d$ wave function ${ }^{15}$ may be responsible for the strength of these peaks and for their energy shift with respect to the $28-\mathrm{eV}$ threshold. Note that the energy positions of these two maxima correspond closely to the energy of the strong $3 p \rightarrow 3 d,{ }^{1} S_{0} \rightarrow{ }^{1} P_{1}$ dipole resonance in both atomic ${ }^{15}$ and metallic ${ }^{16} \mathrm{Ca}$. A purely atomic picture, however, provides no explanation for a splitting of this resonance.

In conclusion, we have presented the dielectric function of $\mathrm{CaF}_{2}$ between 10 and $35 \mathrm{eV}$, which was directly measured by spectroscopic ellipsometry. Close to the fundamental absorption threshold our spectra compare well with the joint density of states from a recent bandstructure calculation ${ }^{8}$ if the energy scale of the calculation is shifted by $1.5 \mathrm{eV}$. At higher energies, the calculated band structure fails to predict the correct critical-point energies but may still serve as a guide to assign the observed structures. At $27.9 \mathrm{eV}$ the $\mathrm{Ca} 3 p$ absorption sets in with a sharp exciton possibly related to conduction-band states at the $X$ point. The structure immediately following is associated with the variation of the density of conduction states. Strong transitions observed in the $32-35-\mathrm{eV}$ region are attributed to $3 p \rightarrow 3 d$ excitations which cannot be well described within the one-electron band-structure formalism.

The work is supported by the Bundesministerium für Forschung und Technologie (BMFT) under Project No. $05490 \mathrm{CAB}$. 
"Present address: Elektro-Optik GmbH, Fördestrasse 35, D2392 Glücksburg, Federal Republic of Germany.

†Present address: II. Institut für Experimentalphysik, Universität Hamburg, Luruper Chaussee 149, D-2000 Hamburg 50, Federal Republic of Germany.

'R. L. Johnson, J. Barth, M. Cardona, D. Fuchs, and A. M. Bradshaw, Rev. Sci. Instrum. 60, 2209 (1989).

${ }^{2}$ T. Tomiki and T. Miyata, J. Phys. Soc. Jpn. 27, 658 (1969).

${ }^{3}$ G. Stephan, Y. LeCalvez, J. C. Lemonnier, and S. Robin, J. Phys. Chem. Solids 30, 601 (1969).

${ }^{4}$ W. Hayes, A. B. Kunz, and E. E. Koch, J. Phys. C 4, L200 (1971).

${ }^{5}$ G. W. Rubloff, Phys. Rev. B 5, 662 (1972).

${ }^{6} J$. Frandon, B. Lahaye, and F. Pradal, Phys. Status Solidi B 53, 565 (1972).

${ }^{7}$ M. Krumrey, E. Tegeler, J. Barth, M. Krisch, F. Schäfers, and R. Wolf, Appl. Opt. 27, 4336 (1988).
${ }^{8}$ R. A. Heaton and C. C. Lin, Phys. Rev. B 22, 3629 (1980).

${ }^{9}$ M. S. Hybertsen and S. G. Louie, Phys. Rev. B 34, 5390 (1986).

${ }^{10}$ M. Alouani, L. Brey, and N. E. Christensen, Phys. Rev. B 37, 1167 (1988).

${ }^{11}$ D. Rieger, F. J. Himpsel, U. O. Karlsson, F. R. McFeely, J. F. Morar, and J. A. Yarmoff, Phys. Rev. B 34, 7295 (1986).

${ }^{12}$ R. T. Poole, J. Szajman, R. C. G. Leckey, J. G. Jenkins, and J. Liesegang, Phys. Rev. B 12, 5872 (1975).

${ }^{13}$ J. Sugar and C. Corliss, J. Phys. Chem. Ref. Data 14, Suppl. 2, 72 (1985).

${ }^{14}$ P. Rabe, B. Sonntag, T. Sagawa, and R. Haensel, Phys. Status Solidi B 50, 559 (1972).

${ }^{15}$ M. W. D. Mansfield and G. H. Newsom, Proc. R. Soc. London Ser. A 357, 77 (1977).

${ }^{16}$ J. Barth, I. Chorkendorff, F. Gerken, C. Kunz, R. Nyholm, J. Schmidt-May, and G. Wendin, Phys. Rev. B 30, 6251 (1984). 